\title{
GRADIENT ESTIMATES AND LIOUVILLE TYPE THEOREMS FOR A NONLINEAR ELLIPTIC EQUATION
}

\author{
GUANGYUE HUANG AND BINGQING MA
}

\begin{abstract}
Let $\left(M^{n}, g\right)$ be an $n$-dimensional complete Riemannian manifold. We consider gradient estimates and Liouville type theorems for positive solutions to the following nonlinear elliptic equation:

$$
\Delta u+a u \log u=0 \text {, }
$$

where $a$ is a nonzero constant. In particular, for $a<0$, we prove that any bounded positive solution of the above equation with a suitable condition for $a$ with respect to the lower bound of Ricci curvature must be $u \equiv 1$. This generalizes a classical result of Yau.
\end{abstract}

\section{INTRODUCTION}

In this paper, we study positive solutions of the equation

$$
\Delta u+a u \log u=0
$$

on an $n$-dimensional complete Riemannian manifold $\left(M^{n}, g\right)$, where $a$ is a nonzero constant. The first study of this and related nonlinear equations can be traced back to Li [6], and later by Ma [7] and Yang [11, who derived various gradient estimates and Harnack estimates and noted the relation to gradient Ricci solitons, which are self-similar solutions to Ricci flow and arise in the blow-up procedure of the long time existence or convergence of the flow. Moreover, the equation (1.1) is closely related to the famous Gross Logarithmic Sobolev inequality, see [4]. For the recent research of (1.1), on can refer to $[2,3,5,8,10$ and the references therein.

It is well-known that for complete noncompact Riemannian manifolds with Ric $\geq 0$, Yau [12] showed that every positive or bounded solution to the equation

$$
\Delta u=0
$$

is constant. We note that the equation (1.2) can be seen as a special case of (1.1) when $a=0$. Therefore, a natural idea is to achieve similar Liouville type theorems for positive solutions to the nonlinear elliptic equation (1.1).

2010 Mathematics Subject Classification. Primary 58J35, Secondary 35B45.

Key words and phrases. Gradient estimate, nonlinear elliptic equation, Liouville-type theorem.

The research of the first author is supported by NSFC(No. 11371018, 11171091). The research of the second author is supported by NSFC (No. 11401179). 
Inspired by the method used by Brighton in [1], this paper is concerned with gradient estimates and Liouville type theorems for positive solutions to the nonlinear elliptic equation (1.1) with $a \neq 0$ and obtain the following results:

Theorem 1.1. Let $\left(M^{n}, g\right)$ be an $n$-dimensional complete Riemannian manifold with $\operatorname{Ric}\left(B_{p}(2 R)\right) \geq-K$, where $K \geq 0$ is a constant. Suppose that $u$ is a positive solution to (1.1) on $B_{p}(2 R)$. Then on $B_{p}(R)$, the following inequalities hold:

(1) If $a>0$, then

$$
|\nabla u| \leq M \sqrt{\frac{(n+3)^{2}}{2 n}(a+K)+\frac{C}{R^{2}}(1+\sqrt{K} R \operatorname{coth}(\sqrt{K} R))} ;
$$

(2) If $a<0$, then

$$
|\nabla u| \leq M \sqrt{\frac{(5 n+6)^{2}}{36 n} \max \{0, a+K\}+\frac{C}{R^{2}}(1+\sqrt{K} R \operatorname{coth}(\sqrt{K} R))},
$$

where $M=\sup _{x \in B_{p}(2 R)} u(x)$ and the constant $C$ depends only on $n$.

Letting $R \rightarrow \infty$, we obtain the following gradient estimates on complete noncompact Riemannian manifolds:

Corollary 1.2. Let $\left(M^{n}, g\right)$ be an n-dimensional complete noncompact Riemannian manifold with $\mathrm{Ric} \geq-K$, where $K \geq 0$ is a constant. Suppose that $u$ is a positive solution to (1.1). Then the following inequalities hold:

(1) If $a>0$, then

$$
|\nabla u| \leq \frac{(n+3) M}{\sqrt{2 n}} \sqrt{a+K}
$$

(2) If $a<0$, then

$$
|\nabla u| \leq \frac{(5 n+6) M}{6 \sqrt{n}} \sqrt{\max \{0, a+K\}}
$$

where $M=\sup _{x \in M^{n}} u(x)$.

In particular, for $a<0$, if $a \leq-K$, then (1.6) shows that $\max \{0, a+K\}=$ 0 and $|\nabla u| \leq 0$ whenever $u$ is a bounded positive solution to (1.1). This implies that $u \equiv 1$ is a constant. Therefore, the following Liouville-type result follows:

Corollary 1.3. Let $\left(M^{n}, g\right)$ be an $n$-dimensional complete noncompact Riemannian manifold with Ric $\geq-K$, where $K \geq 0$ is a constant. Suppose that $u$ is a bounded positive solution defined on $M^{n}$ to (1.1) with $a<0$. If $a \leq-K$, then $u \equiv 1$ is a constant.

In particular, we have the following result: 
Corollary 1.4. Let $\left(M^{n}, g\right)$ be an $n$-dimensional complete noncompact Riemannian manifold with Ric $\geq 0$. Suppose that $u$ is a bounded positive solution defined on $M^{n}$ to (1.1) with $a<0$, then $u \equiv 1$ is a constant.

Remark 1.1. Clearly, our Corollary 1.4 generalizes a uniqueness result of Yau with respect to the heat equation to the nonlinear elliptic equation (1.1) with $a<0$.

Remark 1.2. For an n-dimensional complete noncompact Riemannian manifold with Ric $\geq 0$, it has been shown in Corollary 1.1 of [5] that for positive solutions of (1.1) with $a<0$,

$$
\frac{|\nabla u|^{2}}{u^{2}}+a \alpha \log u \leq \frac{-n a \alpha^{2}}{8}
$$

for any $\alpha>1$. From (1.7), we can not obtain the uniqueness theorem for any bounded positive solution of (1.1). Therefore, our Corollary 1.4 generalizes (2) in Corollary 1.1 of [5] in this sense.

\section{Proof of RESUlts}

The proof of main results will follow from applying the Bochner formula to an appropriate function $h$ of a given positive solution $u$.

Let $h=u^{\epsilon}$, where $\epsilon \neq 0$ is a constant to be determined. Then we have

$$
\log h=\epsilon \log u
$$

A straightforward computation gives

$$
\begin{aligned}
\Delta h & =\Delta\left(u^{\epsilon}\right)=\epsilon(\epsilon-1) u^{\epsilon-2}|\nabla u|^{2}+\epsilon u^{\epsilon-1} \Delta u \\
& =\epsilon(\epsilon-1) u^{\epsilon-2}|\nabla u|^{2}-a \epsilon u^{\epsilon} \log u \\
& =\frac{\epsilon-1}{\epsilon} \frac{|\nabla h|^{2}}{h}-a h \log h .
\end{aligned}
$$

Hence, we have

$$
\begin{aligned}
\nabla h \nabla \Delta h & =\nabla h \nabla\left(\frac{\epsilon-1}{\epsilon} \frac{|\nabla h|^{2}}{h}-a h \log h\right) \\
& =\frac{\epsilon-1}{\epsilon} \nabla h \nabla \frac{|\nabla h|^{2}}{h}-a \nabla h \nabla(h \log h) \\
& =\frac{\epsilon-1}{\epsilon h} \nabla h \nabla\left(|\nabla h|^{2}\right)-\frac{\epsilon-1}{\epsilon} \frac{|\nabla h|^{4}}{h^{2}}-a h \log h \frac{|\nabla h|^{2}}{h}-a|\nabla h|^{2}
\end{aligned}
$$


Applying (2.2) and (2.3) into the well-known Bochner formula to $h$, we have

$$
\begin{aligned}
\frac{1}{2} \Delta|\nabla h|^{2}= & \left|\nabla^{2} h\right|^{2}+\nabla h \nabla \Delta h+\operatorname{Ric}(\nabla h, \nabla h) \\
\geq & \frac{1}{n}(\Delta h)^{2}+\nabla h \nabla \Delta h-K|\nabla h|^{2} \\
= & \frac{1}{n}\left(\frac{\epsilon-1}{\epsilon} \frac{|\nabla h|^{2}}{h}-a h \log h\right)^{2}+\frac{\epsilon-1}{\epsilon} \frac{\nabla h}{h} \nabla\left(|\nabla h|^{2}\right) \\
& -\frac{\epsilon-1}{\epsilon} \frac{|\nabla h|^{4}}{h^{2}}-a h \log h \frac{|\nabla h|^{2}}{h}-(a+K)|\nabla h|^{2} \\
= & \left(\frac{(\epsilon-1)^{2}}{n \epsilon^{2}}-\frac{\epsilon-1}{\epsilon}\right) \frac{|\nabla h|^{4}}{h^{2}}-a\left(\frac{2(\epsilon-1)}{n \epsilon}+1\right) h \log h \frac{|\nabla h|^{2}}{h} \\
& +\frac{a^{2}}{n}(h \log h)^{2}+\frac{\epsilon-1}{\epsilon} \frac{\nabla h}{h} \nabla\left(|\nabla h|^{2}\right)-(a+K)|\nabla h|^{2} .
\end{aligned}
$$

Now we let

$$
a\left(\frac{2(\epsilon-1)}{n \epsilon}+1\right) \geq 0 .
$$

Then for a fixed point $p$, if there exist a positive constant $\delta$ such that $h \log h \leq \delta \frac{|\nabla h|^{2}}{h},(2.4)$ becomes

$$
\begin{aligned}
\frac{1}{2} \Delta|\nabla h|^{2} \geq & {\left[\frac{(\epsilon-1)^{2}}{n \epsilon^{2}}-\frac{\epsilon-1}{\epsilon}-a \delta\left(\frac{2(\epsilon-1)}{n \epsilon}+1\right)\right] \frac{|\nabla h|^{4}}{h^{2}} } \\
& +\frac{a^{2}}{n}(h \log h)^{2}+\frac{\epsilon-1}{\epsilon} \frac{\nabla h}{h} \nabla\left(|\nabla h|^{2}\right)-(a+K)|\nabla h|^{2} \\
\geq & {\left[\frac{(\epsilon-1)^{2}}{n \epsilon^{2}}-\frac{\epsilon-1}{\epsilon}-a \delta\left(\frac{2(\epsilon-1)}{n \epsilon}+1\right)\right] \frac{|\nabla h|^{4}}{h^{2}} } \\
& +\frac{\epsilon-1}{\epsilon} \frac{\nabla h}{h} \nabla\left(|\nabla h|^{2}\right)-(a+K)|\nabla h|^{2} .
\end{aligned}
$$

On the contrary, at the point $p$, if $h \log h \geq \delta \frac{|\nabla h|^{2}}{h}$, then (2.4) becomes

$$
\begin{aligned}
\frac{1}{2} \Delta|\nabla h|^{2} \geq & \left(\frac{(\epsilon-1)^{2}}{n \epsilon^{2}}-\frac{\epsilon-1}{\epsilon}\right) \frac{|\nabla h|^{4}}{h^{2}}+\left[\frac{a^{2}}{n}-\frac{a}{\delta}\left(\frac{2(\epsilon-1)}{n \epsilon}+1\right)\right](h \log h)^{2} \\
& +\frac{\epsilon-1}{\epsilon} \frac{\nabla h}{h} \nabla\left(|\nabla h|^{2}\right)-(a+K)|\nabla h|^{2} \\
\geq & \left.\left(\frac{(\epsilon-1)^{2}}{n \epsilon^{2}}-\frac{\epsilon-1}{\epsilon}\right)+\delta^{2}\left[\frac{a^{2}}{n}-\frac{a}{\delta}\left(\frac{2(\epsilon-1)}{n \epsilon}+1\right)\right]\right\} \frac{|\nabla h|^{4}}{h^{2}} \\
& +\frac{\epsilon-1}{\epsilon} \frac{\nabla h}{h} \nabla\left(|\nabla h|^{2}\right)-(a+K)|\nabla h|^{2} \\
\geq & \left.\left(\frac{(\epsilon-1)^{2}}{n \epsilon^{2}}-\frac{\epsilon-1}{\epsilon}\right)-a \delta\left(\frac{2(\epsilon-1)}{n \epsilon}+1\right)\right\} \frac{|\nabla h|^{4}}{h^{2}} \\
& +\frac{\epsilon-1}{\epsilon} \frac{\nabla h}{h} \nabla\left(|\nabla h|^{2}\right)-(a+K)|\nabla h|^{2}
\end{aligned}
$$


as long as

$$
\frac{a^{2}}{n}-\frac{a}{\delta}\left(\frac{2(\epsilon-1)}{n \epsilon}+1\right)>0 .
$$

In order to obtain the bound of $|\nabla h|$ by using the maximum principle for (2.7), it is sufficient to choose the coefficient of $\frac{|\nabla h|^{4}}{h^{2}}$ in (2.7) is positive, that is,

$$
\left(\frac{(\epsilon-1)^{2}}{n \epsilon^{2}}-\frac{\epsilon-1}{\epsilon}\right)-a \delta\left(\frac{2(\epsilon-1)}{n \epsilon}+1\right)>0 .
$$

We next consider two cases:

Case one: $a>0$.

In this case, provided $\epsilon \in\left(\frac{2}{n+2}, \frac{6}{(5-\sqrt{13}) n+6}\right)$, there will exist an $\delta$ satisfying (2.5), (2.8) and (2.9). In particular, we choose

$$
\epsilon=\frac{3}{n+3}
$$

and

$$
\delta=\frac{n}{2 a},
$$

then (2.7) becomes

$$
\frac{1}{2} \Delta|\nabla h|^{2} \geq \frac{5 n}{18} \frac{|\nabla h|^{4}}{h^{2}}-\frac{n}{3} \frac{\nabla h}{h} \nabla\left(|\nabla h|^{2}\right)-(a+K)|\nabla h|^{2} .
$$

Case two: $a<0$.

In this case, provided $\epsilon \in\left(\frac{6}{(5+\sqrt{13}) n+6}, \frac{2}{n+2}\right)$, there will exist an $\delta$ satisfying (2.5), (2.8) and (2.9). In particular, we choose

$$
\epsilon=\frac{6}{5 n+6}
$$

and

$$
\delta=-\frac{3 n}{4 a}
$$

In particular, in this case, (2.7) becomes

$$
\frac{1}{2} \Delta|\nabla h|^{2} \geq \frac{37 n}{36} \frac{|\nabla h|^{4}}{h^{2}}-\frac{5 n}{6} \frac{\nabla h}{h} \nabla\left(|\nabla h|^{2}\right)-(a+K)|\nabla h|^{2} .
$$

\section{Proof of Theorem 1.1.}

First, we prove the case of $a>0$.

Denote by $B_{p}(R)$ the geodesic ball centered at $p$ with radius $R$. Take a cut-off function $\phi$ (see [9]) satisfying $\operatorname{supp}(\phi) \subset B_{p}(2 R),\left.\phi\right|_{B_{p}(R)}=1$ and

$$
\begin{gathered}
\frac{|\nabla \phi|^{2}}{\phi} \leq \frac{C}{R^{2}} \\
-\Delta \phi \leq \frac{C}{R^{2}}(1+\sqrt{K} R \operatorname{coth}(\sqrt{K} R)),
\end{gathered}
$$

where $C$ is a constant depending only on $n$. Define $G=\phi|\nabla h|^{2}$. Next we will apply maximum principle to $G$ on $B_{p}(2 R)$. Assume $G$ achieves its 
maximum at the point $x_{0} \in B_{p}(2 R)$ and assume $G\left(x_{0}\right)>0$ (otherwise the proof is trivial). Then at the point $x_{0}$, it holds that

$$
\Delta G \leq 0, \quad \nabla\left(|\nabla h|^{2}\right)=-\frac{|\nabla h|^{2}}{\phi} \nabla \phi
$$

and

$$
\begin{aligned}
0 \geq & \Delta G \\
= & \phi \Delta\left(|\nabla h|^{2}\right)+|\nabla h|^{2} \Delta \phi+2 \nabla \phi \nabla|\nabla h|^{2} \\
= & \phi \Delta\left(|\nabla h|^{2}\right)+\frac{\Delta \phi}{\phi} G-2 \frac{|\nabla \phi|^{2}}{\phi^{2}} G \\
\geq & 2 \phi\left[\frac{5 n}{18} \frac{|\nabla h|^{4}}{h^{2}}-\frac{n}{3} \frac{\nabla h}{h} \nabla\left(|\nabla h|^{2}\right)-(a+K)|\nabla h|^{2}\right] \\
& +\frac{\Delta \phi}{\phi} G-2 \frac{|\nabla \phi|^{2}}{\phi^{2}} G \\
= & \frac{5 n}{9} \frac{G^{2}}{\phi h^{2}}+\frac{2 n G}{3 \phi} \nabla \phi \frac{\nabla h}{h}-2(a+K) G \\
& +\frac{\Delta \phi}{\phi} G-2 \frac{|\nabla \phi|^{2}}{\phi^{2}} G,
\end{aligned}
$$

where the second inequality used (2.12). Multiplying both sides of (2.18) by $\frac{\phi}{G}$ yields

$$
\frac{5 n}{9} \frac{G}{h^{2}} \leq-\frac{2 n}{3} \nabla \phi \frac{\nabla h}{h}+2 \phi(a+K)-\Delta \phi+2 \frac{|\nabla \phi|^{2}}{\phi} .
$$

Applying the Cauchy inequality

$$
\begin{aligned}
-\frac{2 n}{3} \nabla \phi \frac{\nabla h}{h} & \leq \frac{2 n}{3}|\nabla \phi| \frac{|\nabla h|}{h} \\
& \leq \frac{n}{3 \varepsilon} \frac{|\nabla \phi|^{2}}{\phi}+\frac{n \varepsilon}{3 h^{2}} \phi|\nabla h|^{2} \\
& =\frac{n}{3 \varepsilon} \frac{|\nabla \phi|^{2}}{\phi}+\frac{n \varepsilon}{3 h^{2}} G,
\end{aligned}
$$

where $\varepsilon \in\left(0, \frac{5}{3}\right)$ is a positive constant, into (2.19) gives

$$
\begin{aligned}
\frac{(5-3 \varepsilon) n}{9} \frac{G}{h^{2}} & \leq 2 \phi(a+K)-\Delta \phi+\left(2+\frac{n}{3 \varepsilon}\right) \frac{|\nabla \phi|^{2}}{\phi} \\
& \leq 2(a+K)-\Delta \phi+\left(2+\frac{n}{3 \varepsilon}\right) \frac{|\nabla \phi|^{2}}{\phi} .
\end{aligned}
$$

In particular, choosing $\varepsilon=\frac{1}{3}$ in (2.20) and applying (2.16) and (2.17), we obtain

$$
\begin{aligned}
\frac{4 n G}{9 h^{2}} & \leq 2(a+K)-\Delta \phi+(n+2) \frac{|\nabla \phi|^{2}}{\phi} \\
& \leq 2(a+K)+\frac{C}{R^{2}}(1+\sqrt{K} \operatorname{coth}(\sqrt{K} R)) .
\end{aligned}
$$


It follows that for $x \in B_{p}(R)$,

$$
\begin{aligned}
\frac{4 n}{9} G(x) & \leq \frac{4 n}{9} G\left(x_{0}\right) \\
& \leq h^{2}\left(x_{0}\right)\left[\frac{(n+3)^{2}}{2 n}(a+K)+\frac{C}{R^{2}}(1+\sqrt{K} R \operatorname{coth}(\sqrt{K} R))\right] .
\end{aligned}
$$

This shows

$$
|\nabla u|^{2}(x) \leq M^{2}\left[\frac{(n+3)^{2}}{2 n}(a+K)+\frac{C}{R^{2}}(1+\sqrt{K} R \operatorname{coth}(\sqrt{K} R))\right]
$$

and

$$
|\nabla u| \leq M \sqrt{\frac{(n+3)^{2}}{2 n}(a+K)+\frac{C}{R^{2}}(1+\sqrt{K} R \operatorname{coth}(\sqrt{K} R))},
$$

where $M=\sup _{x \in B_{p}(2 R)} u(x)$. This yields the desired inequality (1.3) of Theorem 1.1 .

Next, we prove the case of $a<0$.

Define $\tilde{G}=\phi|\nabla h|^{2}$. We apply maximum principle to $\tilde{G}$ on $B_{p}(2 R)$. Assume $\tilde{G}$ achieves its maximum at the point $\tilde{x}_{0} \in B_{p}(2 R)$ and assume $\tilde{G}\left(\tilde{x}_{0}\right)>0$ (otherwise the proof is trivial). Then at the point $\tilde{x}_{0}$, it holds that

$$
\Delta \tilde{G} \leq 0, \quad \nabla\left(|\nabla h|^{2}\right)=-\frac{|\nabla h|^{2}}{\phi} \nabla \phi
$$

and

$$
\begin{aligned}
0 \geq & \Delta \tilde{G} \\
= & \phi \Delta\left(|\nabla h|^{2}\right)+\frac{\Delta \phi}{\phi} \tilde{G}-2 \frac{|\nabla \phi|^{2}}{\phi^{2}} \tilde{G} \\
\geq & 2 \phi\left[\frac{37 n}{36} \frac{|\nabla h|^{4}}{h^{2}}-\frac{5 n}{6} \frac{\nabla h}{h} \nabla\left(|\nabla h|^{2}\right)-(a+K)|\nabla h|^{2}\right] \\
& +\frac{\Delta \phi}{\phi} \tilde{G}-2 \frac{|\nabla \phi|^{2}}{\phi^{2}} \tilde{G} \\
= & \frac{37 n}{18} \frac{\tilde{G}^{2}}{\phi h^{2}}+\frac{5 n \tilde{G}}{3 \phi} \nabla \phi \frac{\nabla h}{h}-2(a+K) \tilde{G} \\
& +\frac{\Delta \phi}{\phi} \tilde{G}-2 \frac{|\nabla \phi|^{2}}{\phi^{2}} \tilde{G},
\end{aligned}
$$

where the second inequality used (2.15). Multiplying both sides of (2.25) by $\frac{\phi}{\tilde{G}}$ yields

$$
\frac{37 n}{18} \frac{\tilde{G}}{h^{2}} \leq-\frac{5 n}{3} \nabla \phi \frac{\nabla h}{h}+2 \phi(a+K)-\Delta \phi+2 \frac{|\nabla \phi|^{2}}{\phi} .
$$


Inserting the Cauchy inequality

$$
\begin{aligned}
-\frac{5 n}{3} \nabla \phi \frac{\nabla h}{h} & \leq \frac{5 n}{3}|\nabla \phi| \frac{|\nabla h|}{h} \\
& \leq \frac{5 n}{6 \varepsilon} \frac{|\nabla \phi|^{2}}{\phi}+\frac{5 n \varepsilon}{6 h^{2}} \tilde{G},
\end{aligned}
$$

where $\varepsilon \in\left(0, \frac{37}{15}\right)$ is a positive constant, into (2.26) gives

$$
\begin{aligned}
\frac{(37-15 \varepsilon) n}{18} \frac{\tilde{G}}{h^{2}} & \leq 2 \phi(a+K)-\Delta \phi+\left(2+\frac{5 n}{6 \varepsilon}\right) \frac{|\nabla \phi|^{2}}{\phi} \\
& \leq 2 \max \{0, a+K\}-\Delta \phi+\left(2+\frac{5 n}{6 \varepsilon}\right) \frac{|\nabla \phi|^{2}}{\phi} .
\end{aligned}
$$

Hence, choosing $\varepsilon=\frac{1}{15}$ in (2.20) and applying (2.16) and (2.17), we have

$$
\frac{2 n \tilde{G}}{h^{2}} \leq 2 \max \{0, a+K\}+\frac{C}{R^{2}}(1+\sqrt{K} \operatorname{coth}(\sqrt{K} R)) .
$$

Finally, restricting to $B_{p}(R)$ yields

$$
|\nabla u|^{2} \leq M^{2}\left[\frac{(5 n+6)^{2}}{36 n} \max \{0, a+K\}+\frac{C}{R^{2}}(1+\sqrt{K} R \operatorname{coth}(\sqrt{K} R))\right] .
$$

This concludes the proof of inequality (1.4) of Theorem 1.1

\section{REFERENCES}

[1] K. Brighton, A Liouville-type theorem for smooth metric measure spaces, J. Geom. Anal. (2013) 23: 562-570.

[2] X. D. Cao, B. F. Ljungberg, B. W. Liu, Differential Harnack estimates for a nonlinear heat equation, J. Funct. Anal. (2013) 265: 2312-2330.

[3] L. Chen, W. Y. Chen, Gradient estimates for a nonlinear parabolic equation on complete non-compact Riemannian manifolds, Ann. Glob. Anal. Geom. (2009) 35: 397-404.

[4] L. Gross, Logarithmic Sobolev inequality and contractivity properties of semigroups, in: Dirichlet Forms, Varenna, 1992, in: Lecture Notes in Math., vol. 1563, Springer, Berlin, 1993, pp. 54-88.

[5] G. Y. Huang, Z. J. Huang, H. Z. Li, Gradient estimates and differential Harnack inequalities for a nonlinear parabolic equation on Riemannian manifolds, Ann. Glob. Anal. Geom. (2013) 43: 209-232.

[6] J. Y. Li, Gradient estimates and Harnack inequalities for nonlinear parabolic and nonlinear elliptic equations on Riemannian manifolds, J. Funct. Anal. (1991) 100: 233-256.

[7] L. Ma, Gradient estimates for a simple elliptic equation on complete non-compact Riemannian manifolds, J. Funct. Anal. (2006) 241: 374-382.

[8] B. Qian, A uniform bound for the solutions to a simple nonlinear equation on Riemannian manifolds, Nonlinear Anal. (2010) 73: 1538-1542.

[9] R. Schoen, S.-T. Yau, Lectures on Differential Geometry, International Press, 1994.

[10] J. Y. Wu, Li-Yau type estimates for a nonlinear parabolic equation on complete manifolds, J. Math. Anal. Appl. (2010) 369: 400-407.

[11] Y. Y. Yang, Gradient estimates for a nonlinear parabolic equation on Riemannian manifolds, Proc. Amer. Math. Soc. (2008) 136: 4095-4102. 
[12] S. T. Yau, Harmonic functions on complete Riemannian manifolds, Comm. Pure Appl. Math. (1975) 28: 201-228.

Department of Mathematics, Henan Normal University, XinXiang 453007, P.R. China

E-mail address: hgy@henannu.edu.cn

Department of Mathematics, Henan Normal University, Xinxiang 453007, P.R. China

E-mail address: bqma@henannu.edu.cn 